\title{
Intrauterine retention of fetal bones, a cause for secondary infertility
}

Anil Humane, Nikita Agrawal*

Department of Obstetrics and Gynaecology, Government Medical College Nagpur, Maharashtra, India

Received: 03 August 2019

Accepted: 13 September 2019

\section{*Correspondence:}

Dr. Nikita Agrawal,

E-mail: nikitaagrawal286@gmail.com

Copyright: (C) the author(s), publisher and licensee Medip Academy. This is an open-access article distributed under the terms of the Creative Commons Attribution Non-Commercial License, which permits unrestricted non-commercial use, distribution, and reproduction in any medium, provided the original work is properly cited.

\section{ABSTRACT}

Secondary infertility due to retained products of conception in the form of fetal bones is a rare entity. It is usually associated with a history of abortion, either spontaneous or induced. The bony fragments probably act as an intrauterine contraceptive device to stimulate the secretion of endometrial prostaglandins, resulting in secondary infertility. The common symptoms include menstrual disturbances, pelvic pain, dyspareunia, vaginal discharge, spontaneous passage of fetal bones and infertility. Advanced diagnostic technique like hysteroscopy has helped in the diagnosis.

Keywords: Abortion, Chronic pelvic pain, Hypomenorrhoea, Hysteroscopy, Intrauterine fetal bones, Secondary infertility

\section{INTRODUCTION}

Secondary infertility due to retained products of conception in the form of fetal bones is a rare entity, and the bony fragments probably act as an intrauterine contraceptive device to stimulate the secretion of endometrial prostaglandins, resulting in secondary infertility. It is usually associated with a history of abortion, either spontaneous or induced. ${ }^{1}$ The common symptoms include menstrual disturbances, pelvic pain, dyspareunia, vaginal discharge, spontaneous passage of fetal bones and infertility. ${ }^{2}$ The most widely accepted theory is that the endometrial ossification represents retained fetal bones following spontaneous, missed, incomplete or therapeutic abortion. Cases are diagnosed by transvaginal ultrasound, hysterosalpingogram and in particular, hysteroscopy. ${ }^{3-5}$ Authors present one such case of secondary infertility with history of antecedent abortion with retained fetal bones in the uterine cavity. The role of hysteroscopy has also been emphasized in the diagnosis and management of this case.

\section{CASE REPORT}

A 29 years old female P1L1A1, married since 9 years, reported to GMCH OPD with complaints of inability to conceive since 1 year, chronic pelvic pain and hypomenorrhoea. She had a history of full term lscs delivery 5 years back i/v/o breech presentation followed by MTP at 4 months of gestation 1 year back i/v/o fetal anomaly. Her menstrual cycle was $1 / 30$ days, scanty menses. Previous cycles were 3/30, regular with moderate flow. There was no history suggestive of tuberculosis, thyroid disorder. No significant past/personal/family history. General and systemic examination was within normal limits. Abdomen is soft nontender, no palpable mass present. On per vaginal examination- uterus normal size, anteverted, b/l fornices free, nontender.

Patient was diagnosed and investigated as a case of secondary infertility. Routine blood investigations, Husband's semen analysis was within normal limits. USG 
showed features s/o? Tubercular endometritis as endometrium is hyperechoic. She was subjected to diagnostic hysterolaparoscopy. Hysteroscopy showed presence of multiple retained fetal bones (Figure 1) with trabeculae, embedded in the endometrium (Figure 2).

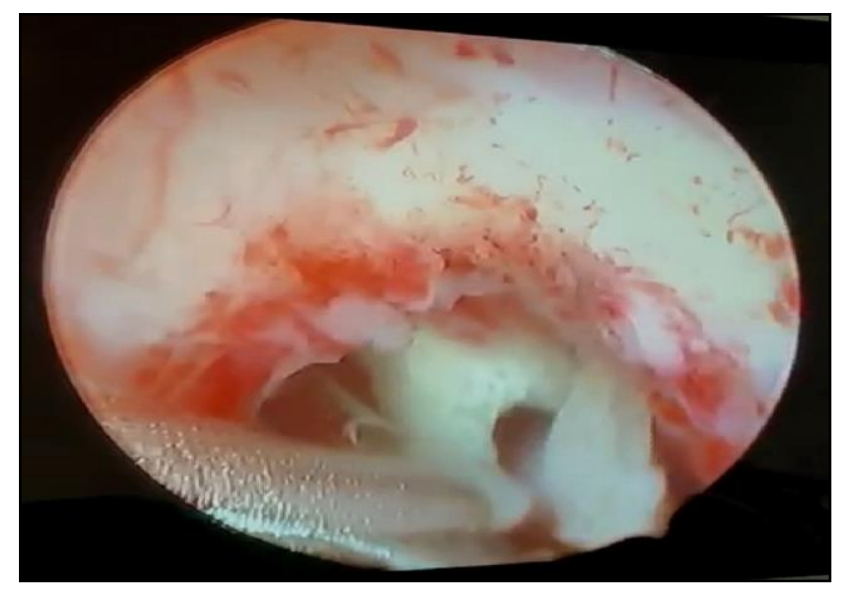

Figure 1: Hysteroscopic view of retained intrauterine fetal bones.

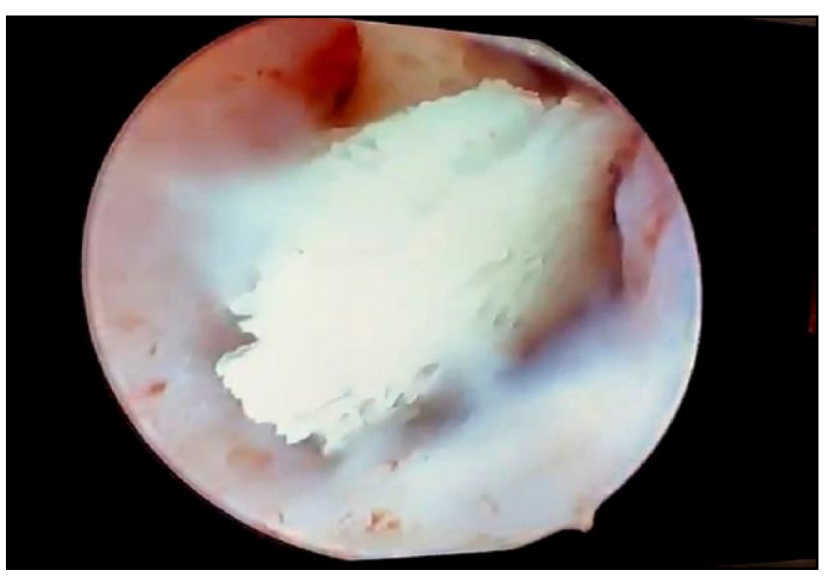

Figure 2: Hysteroscopic view showing trabecular pattern of fetal bones.

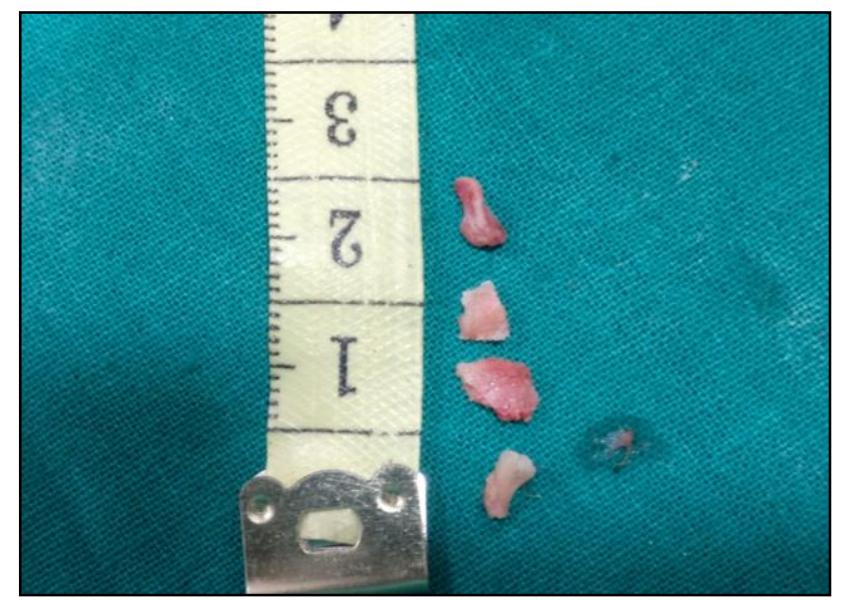

Figure 3: Naked view of bone fragments removed hysteroscopically.
Fetal Bones were removed hysteroscopically at the same sitting. Figure 3 shows naked view appearance of fetal bones, which were removed hysteroscopically. Laparoscopy showed normal uterus, ovaries and fallopian tubes with normal patency. Post-operative period was uneventful. Patient was discharged on tab Premarin for 3 months. On follow up evaluation after 2 months, TVS showed normal endometrial thickness, no retained fetal bones and no adhesions. Patient now has normal cycles and is undergoing treatment for infertility.

\section{DISCUSSION}

Every year many abortions are performed. Although abortion is an extremely safe procedure, complications do occur. This case illustrates a rare cause of secondary infertility due to the prolonged retention of fetal bones. Intrauterine retained fetal bones represents a rare cause of secondary infertility, hypomenorrhoea and chronic pelvic pain. On reviewing literature worldwide, very few such cases have been documented. With the advent of newer techniques like hysteroscopy, more cases are being reported. Waxman M and Missouris HF have reported a case of endometrial calcification following spontaneous abortion. ${ }^{6}$

In 1991, Melius et al, have also reported two cases of prolonged intrauterine retention of fetal bones. ${ }^{7}$ Dawood and Tarrett postulated that these structures act like an intrauterine contraceptive devices thus preventing implantation. ${ }^{8}$ The diagnosis of retained fetal bone can be made by identifying a filling defect on a hysterosalpingogram, an echogenic area on vaginal ultrasound or, as in our case, by direct visualization on hysteroscopy. Hysteroscopy remains the investigation of choice for diagnosis of such intrauterine pathology and treatment at the same time. ${ }^{9}$ It is quite possible that such cases were being missed earlier due to lack of such facilities. Furthermore, fertility can be increased following removal of the fetal bones.

\section{CONCLUSION}

This case report stresses the importance of taking a thorough history taking and evaluation of the endometrium by transvaginal ultrasound or hysteroscopy in women with secondary infertility. The purpose of reporting this case was to bring to light a rare cause of infertility and the role of hysteroscopy in its management. A high success rate may be expected following the removal of any retained bones. This case highlights the importance of careful examination of intactness of removed fetus and review USG following $2^{\text {nd }}$ trimester abortions for timely detection of any potential abnormalities.

Funding: No funding sources Conflict of interest: None declared Ethical approval: Not required 


\section{REFERENCES}

1. Basu M, Mammen C, Owen E. Bony fragments in the uterus: an association with secondary subfertility. Ultrasound Obstet Gynecol. 2003;22(4):402-6.

2. Graham O, Cheng LC, Parsons JH. The ultrasound diagnosis of retained foetal bones in West African patients complaining of infertility. BJOG. 2000;107(1):122-4.

3. Elford K, Claman P. Novel treatment of a patient with secondary infertility due to retained fetal bone. Fertil Steril. 2003;79(4):1028-30.

4. Moon HS, Park YH, Kwon HY, Hong SH, Kim SK. Iatrogenic secondary infertility caused by residual intrauterine fetal bone after midtrimester abortion. Am J Obstet Gynecol. 1997;176:369-70.

5. Tulandi T, Sammour A. Retained fetal bones in the uterine cavity. J Am Assoc Gynecol Laparosc. 2001;8:179-80.
6. Waxman M, Missouris HF. Endometrial ossification following abortion. Amer J Obst Gyn. 1978;130:587-9.

7. Melius FA, Thomas MJ, Theodore CN. Prolonged retention of intrauterine bones. Obst Gynec. 1991;78:919-20.

8. Dawood YM. Tarret JC III. Prolonged intrauterine retention of foetal bones after abortion causing infertility. Am J Obstet Gynec. 1982;143:715-8.

9. Aranmja U, Preron SB, Pankin OF. Osseous metaplasia of endometrium treated by hysteroscopic resection. Br J Obst Gynecol. 1993;100:391-2.

Cite this article as: Humane A, Agrawal N. Intrauterine retention of fetal bones, a cause for secondary infertility. Int J Reprod Contracept Obstet Gynecol 2019;8:4101-3. 